\title{
Invariance Principle for Wave Propagation inside Inhomogeneously Disordered Materials
}

\author{
Yiming Huang, ${ }^{1, *}$ Chushun Tian, ${ }^{2, *}$ Victor A. Gopar, ${ }^{3, \dagger}$ Ping Fang, ${ }^{4}$ and Azriel Z. Genack ${ }^{1, \$}$ \\ ${ }^{1}$ Department of Physics, Queens College and The Graduate Center of the City University of New York, Flushing, New York 11367, USA \\ ${ }^{2}$ CAS Key Laboratory of Theoretical Physics and Institute of Theoretical Physics, Chinese Academy of Sciences, Beijing 100190, China \\ ${ }^{3}$ Departamento de Física Teórica and BIFI, Universidad de Zaragoza, Pedro Cerbuna 12, 50009 Zaragoza, Spain \\ ${ }^{4}$ School of Science, Beijing University of Posts and Telecommunications, Beijing 100876, China
}

(Received 15 August 2019; accepted 19 December 2019; published 5 February 2020)

\begin{abstract}
Disorder is more the rule than the exception in natural and synthetic materials. Nonetheless, wave propagation within inhomogeneously disordered materials has received scant attention. We combine microwave experiments and theory to find the spatial variation of generic wave propagation quantities in inhomogeneously disordered materials. We demonstrate that wave statistics within samples of any dimension are independent of the detailed structure of a material and depend only on the net strengths of distributed scattering and reflection between the observation point and each of the boundaries.
\end{abstract}

DOI: 10.1103/PhysRevLett.124.057401

Coherent waves launched into a random sample are scrambled in a haze of scattered fields that is manifested in the speckle pattern of scattered light. This makes disordered materials opaque and impairs communication, imaging, and transport. Studies of transport in random systems have focused on disordered materials with uniform scattering strength throughout the medium and possible surface reflection [1-8]. For example, Brownian motion of particles in a homogeneous liquid was shown by Einstein to be due to randomly fluctuating forces on microparticles by molecules in thermal equilibrium [9], while, in Chandrahekar's radiative transfer model, the rate of particle scattering out of or into a particular direction is homogeneous [10]. In quantum scattering, the suppression of electron diffusion was first studied in the Anderson tightbinding model, in which the distributions of electron energy at each lattice site are the same [11]. Localization of radio waves was first found in calculations for random waveguides with a spatially homogeneous distribution of disorder in the dielectric constant [12]. Such studies have led to a deep understanding of ballistic and diffusive propagation and of Anderson localization. However, the paradigm of homogeneously disordered materials does not represent key elements of our surroundings, which are generally inhomogeneously disordered with a spatially varying scattering strength and multiple interfaces within the bulk of the material and at the surface. Understanding wave propagation in such materials would enable a wide range of applications in diverse fields, including medical imaging [13], lidar and remote sensing [14], astrophysics [10], telecommunication [15], electronics [16,17], phononics [18-20], invisibility cloaking [21], and photothermal therapy [22].

In this Letter, we go beyond canonical homogeneously disordered materials. We begin by considering a simple departure from the traditional uniformly disordered material - the insertion of a single reflector into a uniformly disordered sample. We discover an invariance principle for such materials: wave statistics at any point of observation is invariant with regard to displacement of a reflector along the length of the sample, apart from a discontinuity that arises when the reflector crosses that point. This leads to the discovery of an even broader invariance principle. By that principle we obtain a full description of wave propagation and statistics for general inhomogeneously disordered materials with multiple embedded reflectors or tunneling barriers and a longitudinally varying transport mean free path $\ell(x)$.

We carry out microwave measurements in a uniformly disordered one-dimensional (1D) open medium of length $L$ with a reflector at depth $x_{0}$. We consider the intensity at a point $x, I\left(x ; x_{0}\right)$, which is normalized so that its value at the output is equal to the flux transmission coefficient for an ensemble of disorder configurations. The results of measurements of the ensemble averages $\left\langle\ln I\left(x ; x_{0}\right)\right\rangle,\left\langle I\left(x ; x_{0}\right)\right\rangle$ and the probability density function $P\left(\ln I\left(x ; x_{0}\right)\right)$ are explained using random matrix theory (RMT) [5,23,24] and supersymmetry field theory (SUSY) [25,26]. We consider $P(\ln I)$ rather than $P(I)$ since $\ln I$ self-averages so that a comparison to theory can be made with measurements on fewer disorder configurations. RMT and SUSY address wave propagation from different perspectives and together provide a universal description for it.

We find that the average and the probability density function of all local quantities that can be expressed in terms of the Green function of the wave equation share a common feature with regard to their variation with $x_{0}$ : when $x$ is fixed, but $x_{0}$ is changed, a quantity $O\left(x ; x_{0}\right)$ representative of waves in a disordered ensemble is unchanged as long as $x$ remains in front of or behind the reflector, and changes discontinuously from $O(x ; 0)$ to 
$O(x ; L)$ when $x_{0}$ crosses $x$. This behavior is encapsulated in the following identity proved below:

$$
O\left(x ; x_{0}\right)=\theta\left(x_{0}-x\right) O(x ; L)+\theta\left(x-x_{0}\right) O(x ; 0),
$$

where $\theta$ is the Heaviside function. This allows us to find the spatial structure of the statistics of intensity and of the intensity in transmission eigenchannels [27-31]. The impact of distributed scattering and discrete reflection on the profile of $\langle\ln I\rangle$ is additive, while the profile of $\langle I\rangle$ is determined by the position-dependent diffusion coefficient $D\left(x ; x_{0}\right)$, which is a function of the strength of distributed scattering, discrete reflection, and the relative positions of $x$ and $x_{0} . D\left(x ; x_{0}\right)$ not only satisfies Eq. (1), but also obeys a scaling law,

$$
D\left(x ; x_{0}\right) / D_{0}=D_{\infty}\left(\lambda\left(x ; x_{0}\right)\right),
$$

once it is rescaled by the Boltzmann diffusion coefficient $D_{0}$. Here $x$ and all parameters describing the inhomogeneity enter into the scaling factor $\lambda\left(x ; x_{0}\right)$, and the scaling function $D_{\infty}(\lambda)$ describes propagation in both homogeneously and inhomogeneously disordered materials. These findings go beyond traditional studies that address the interplay between surface reflection and particle scattering, which ignore wave interference $[3,10,32-35]$. The results are surprising since the field at $x$ results from the coherent superposition of waves arriving at $x$, which is inevitably affected by the specific location of a reflector, $x_{0}$, placed in front of or behind $x$. Finally, the principle applies not only in $1 \mathrm{D}$, but also in quasi-1D and higher dimensions.

Measurements are carried out with the use of a vector network analyzer in a single-mode rectangular copper waveguide containing ceramic slabs of thickness $6.6 \mathrm{~mm}$ and Teflon U-channel air spacers with thickness randomly chosen from three values: $1.27,2.55$, and $3.82 \mathrm{~cm}$. The sample of length $L=86.0 \mathrm{~cm}$ is contained in a copper waveguide with a cutoff frequency of $6.56 \mathrm{GHz}$. Successive sample elements are selected randomly with a probability of $1 / 2$ for the ceramic slabs and $1 / 6$ for each thickness of the U-channel elements. Spectra are taken for ensembles of
100 random configurations without a reflector and with a reflector placed at relative depths of $x_{0} / L=0,1 / 4,3 / 4$, and 1 . The reflector is a thin copper plate covering $76 \%$ of the waveguide cross section with transmission coefficient $\Gamma=0.36$ in the empty waveguide.

The wave is detected by an antenna inserted sequentially into a series of holes of diameter $3.17 \mathrm{~mm}$ spaced by $1 \mathrm{~cm}$ along the waveguide. Field spectra are taken over the frequency interval $10.00-10.70 \mathrm{GHz}$ in which the waveguide supports a single mode. The incident intensity $I_{0}$ is found by fitting the expression for the intensity of counterpropagating waves: $I_{0}\left[1+r^{2}+2 r \cos (2 k x+\varphi)\right]$ in a 4 -cm-long segment before the random sample to determine the incident field. Here $r$ is the magnitude of the reflected field, $k$ is the wave number determined from waveguide dispersion, and $\varphi$ is the phase. We undo the impact of absorption using the method described in the Supplemental Material [36]. Measurements of intensity at each position are normalized by measurements in the empty waveguide with absorbers at its ends so that the sensitivity is uniform at all positions.

Measured profiles of $\left\langle\ln I\left(x ; x_{0}\right)\right\rangle$ for samples without a reflector and with a reflector at $x_{0}=L / 4$ or $3 L / 4$, in which the impact of absorption is removed are plotted in Fig. 1(a). $\langle\ln I\rangle$ decreases linearly in front of the reflector and overlaps the profile measured in samples without a reflector, but it drops sharply behind the reflector and then continues to fall with the same slope as before the reflector. The profiles in samples with a reflector at $x_{0}=L / 4$ and $3 L / 4$ are identical in the region in front of $(x<L / 4)$ and behind $(x>3 L / 4)$ the reflectors, demonstrating that the size of the drop is independent of $x_{0}$. Away from $x_{0}$, the slope of $\langle\ln I\rangle$ in all regions is the same as the slope in a sample without a reflector. When $x$ is rescaled by $L$, this gives a slope of magnitude $s=L / \ell=3.51$ [24] corresponding to a mean free path $\ell=24.5 \mathrm{~cm}$. In the following and in the Supplemental Material [36], we use RMT to show

$$
\left\langle\ln I\left(x ; x_{0}\right)\right\rangle=\left\{\begin{array}{ll}
-x / \ell & \text { for } x<x_{0} \\
-x / \ell+\ln \Gamma & \text { for } x>x_{0}
\end{array} .\right.
$$
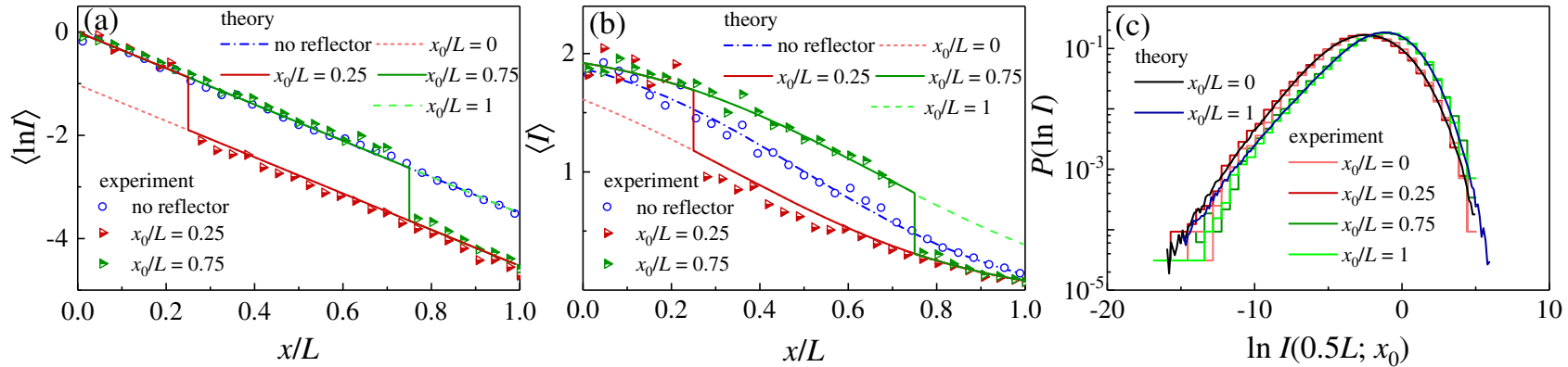

FIG. 1. Measurements of and theoretical results for $O\left(x ; x_{0}\right)=(a)\left\langle\ln I\left(x ; x_{0}\right)\right\rangle$, and (b) $\left\langle I\left(x ; x_{0}\right)\right\rangle$, and (c) $P\left(\ln I\left(0.5 L ; x_{0}\right)\right)$ in $1 \mathrm{D}$ samples with a reflector at different $x_{0}$ or without a reflector are in good agreement. They all exhibit the invariance and discontinuity with regard to displacement of the reflector, and are identical to $O\left((x ; L(0))\right.$ for $x<x_{0}\left(x>x_{0}\right)$. 
The discontinuity at $x_{0}$ is equal to the logarithm of the transmission coefficient $\Gamma$ of the reflector. This analytic result is plotted in Fig. 1(a), and seen to be in excellent agreement with measurements. Equation (3) is a special case of the general result of Eq. (1). For $x<x_{0}\left(x>x_{0}\right)$, $\left\langle\ln I\left(x ; x_{0}\right)\right\rangle$ is the same as when the reflector is at the output (input).

Measured profiles of $\left\langle I\left(x ; x_{0}\right)\right\rangle$ for samples without a reflector and with a reflector at $x_{0}=L / 4$ or $3 L / 4$ are plotted in Fig. 1(b). The profiles for the reflector at $x_{0}=$ $L / 4$ and $3 L / 4$ still overlap for $x<L / 4$ and $x>3 L / 4$, as is the case for $\langle\ln I\rangle$. Though $\left\langle\ln I\left(x ; x_{0}\right)\right\rangle$ in front of the reflectors $(x<L / 4)$ is not affected by the presence of the reflector, $\left\langle I\left(x ; x_{0}\right)\right\rangle$ increases significantly for $x<L / 4$ relative to the profile for samples without a reflector. Using SUSY, we show this is another application of Eq. (1),

$$
\left\langle I\left(x ; x_{0}\right)\right\rangle=\theta\left(x_{0}-x\right)\langle I(x ; L)\rangle+\theta\left(x-x_{0}\right)\langle I(x ; 0)\rangle,
$$

where

$$
\begin{aligned}
\langle I(x ; 0(L))\rangle= & \langle I(0 ; 0(L))\rangle-[\langle I(0 ; 0)\rangle+\langle I(0 ; L)\rangle-2] \\
\times & \frac{\operatorname{erf}\left(\sqrt{\frac{s L}{L+\zeta}} \frac{2 x-(L \mp \zeta)}{2 L}\right)-\operatorname{erf}\left(-\sqrt{\frac{s L}{L+\zeta}} \frac{L \mp \zeta}{2 L}\right)}{\operatorname{erf}\left(\sqrt{\frac{s L}{L+\zeta}} \frac{L \pm \zeta}{2 L}\right)-\operatorname{erf}\left(-\sqrt{\frac{s L}{L+\zeta}} \frac{L \mp \zeta}{2 L}\right)} .
\end{aligned}
$$

Here $\zeta$ is a length determined by $\Gamma$ and $\langle I(0 ; 0)\rangle \equiv$ $\left\langle I\left(0^{+} ; 0\right)\right\rangle$. From Eq. (5), we find $\langle I(x ; 0)\rangle+\langle I(L-x ; L)\rangle=$ 2 . This is in accordance with the sum of intensity at a point due to all incident channels being equal to the local density of states with its average unaffected by the presence of a reflector. Good agreement of Eqs. (4) and (5) with measurements is found for $s=3.51, \zeta=0.33 L$ [36], $\langle I(0 ; 0)\rangle=1.61$, and $\langle I(0 ; L)\rangle=1.92$, with the last two parameters calculated using Eq. (10) below. For samples without a reflector, $\zeta$ vanishes. In this case, we find with the use of Eq. (10) that $\langle I(0 ; 0)\rangle=\langle I(0 ; L)\rangle=1.86$. Substituting this into Eq. (5) gives $\langle I(x ; 0)\rangle=\langle I(x ; L)\rangle$ with a profile in good agreement with measurements.

Measurements of $P\left(\ln I\left(L / 2 ; x_{0}\right)\right)$ at $x_{0} / L=0,1 / 4$, $3 / 4$, and 1 are shown in Fig. 1(c) and compared to RMT calculations for $P(\ln I(L / 2 ; 0(L)))$. We see that the measurements of $P(\ln I)$ for $x_{0} / L=0,1 / 4$ coincide with the theoretical result for $P(\ln I(L / 2 ; 0))$, while, for $x_{0} / L=1,3 / 4$, the measurements match the theoretical result for $P(\ln I(L / 2 ; L))$. This is in accordance with Eq. (1), which predicts that $P\left(\ln I\left(L / 2 ; x_{0}\right)\right)$ is invariant with regard to the displacement of the reflector as long as $\theta\left(x-x_{0}\right)$ does not change, but changes suddenly when $x_{0}$ crosses $L / 2$.

To study universal aspects of $\langle I\rangle$, we show below that

$$
-\partial_{x} D\left(x ; x_{0}\right) \partial_{x}\langle I\rangle=0
$$

in the interior of the medium. This differs from the standard diffusion equation in the position dependence of the diffusion coefficient, which is the result of the spatial variation of localization effects in open media [25,26,51-53].

$$
\begin{aligned}
& \frac{D\left(x ; x_{0}\right)}{D_{0}}=e^{-\lambda\left(x ; x_{0}\right)}, \\
& \lambda\left(x ; x_{0}\right)= \begin{cases}\frac{(x+\zeta)(L-x)}{\ell(L+\zeta)}, & x>x_{0} \\
\frac{x(L+\zeta-x)}{\ell(L+\zeta)}, & x<x_{0}\end{cases}
\end{aligned}
$$

where $\lambda$ is essentially the probability density of return obtained from the diffusion equation with diffusion coefficient $D_{0}$. Because $\lambda$ for $x>x_{0}\left(x<x_{0}\right)$ is the same as when $x_{0}=0(L)$, it obeys Eq. (1), and so does $D$.

Intensity fluctuations are too large to allow for an accurate experimental determination of $D\left(x ; x_{0}\right)$ with data from 100 configurations. Thus we perform simulations using the experimental values for $\ell, L$, and $\Gamma$, and compute $D\left(x ; x_{0}\right)$ from $-\langle T\rangle / \partial_{x}\langle I\rangle$, which is the generalized Fick's law. Here $\langle T\rangle$ is the ensemble-averaged transmission coefficient, which is equal to the flux. Simulation results for $x_{0}=L / 4$ and $3 L / 4$ are shown in Fig. 2(a) and are in good agreement with the analytic result of Eq. (7). We next use the expression for $\lambda$ given above to map $x$ to $\lambda\left(x ; x_{0}\right)$. For samples without a reflector, we set $\zeta=0$ so that $\lambda=x(L-x) /(\ell L)$. The simulated profile $D\left(x ; x_{0}\right) / D_{0}$ for samples with different $x_{0}$ or for samples without a reflector collapse to a single curve: $D_{\infty}(\lambda)=e^{-\lambda}$, as shown in Fig. 2(b). This scaling law was found previously for uniformly disordered materials [26].

We sketch the analytic derivations. The full theory is provided in a self-contained manner in the Supplemental Material [36]. We first consider quasi-1D but locally twodimensional (2D) random media and then extrapolate the final results from quasi-1D to 1D. The dielectric constant $\epsilon(\boldsymbol{r})$ exhibits independent Gaussian fluctuations around unity at every point $\boldsymbol{r} \equiv(x, y)$. The reflector is modeled by
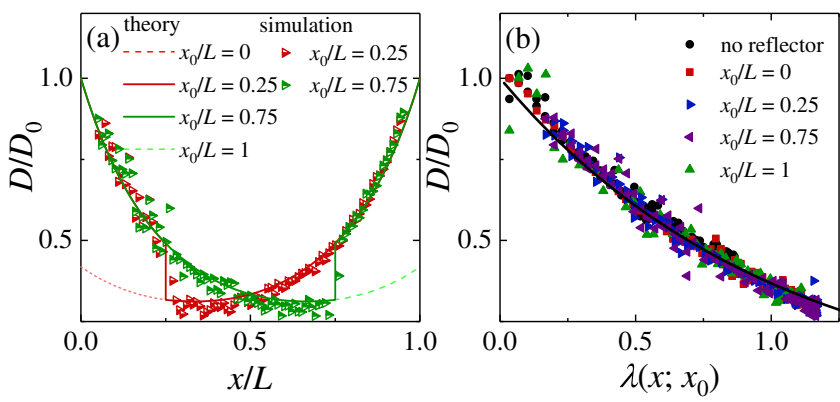

FIG. 2. (a) Simulation and analytic results for $1 \mathrm{D}$ samples with a reflector at different $x_{0}$ show that the profiles $D\left(x ; x_{0}\right)$ overlap $D(x ; L(0))$ for $x<x_{0}\left(x>x_{0}\right)$ and display a discontinuity at $x_{0}$. (b) Upon mapping $x$ to $\lambda\left(x ; x_{0}\right)$, simulated profiles of $D\left(x ; x_{0}\right) / D_{0}$ (symbols) for samples with different $x_{0}$ or without a reflector collapse to a single curve: $D_{\infty}(\lambda)=e^{-\lambda}$ (solid line). 

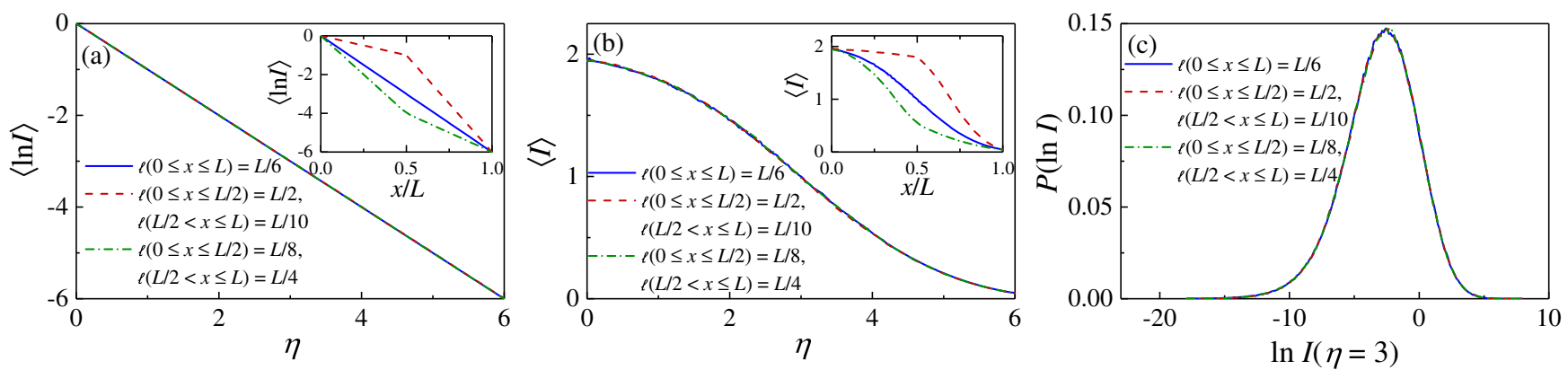

FIG. 3. Simulations for three ensembles, two consisting of 1D samples composed of two segments of different scattering strength (red dashed and green dash-dotted curves) and one of uniformly disordered samples (blue solid curve). In all samples, $\eta_{L}=6$. Upon mapping $x$ to $\eta(x)$, the profiles of (a, inset) $\langle\ln I\rangle$, (b, inset) $\langle I\rangle$, and (c) $P(\ln I)$ in different ensembles collapse to a single curve (main panel).

a layer of high dielectric constant. The propagation of a wave of angular frequency $\omega$ is described by the retarded (advanced) Green function $G^{R(A)} \equiv\left[\nabla^{2}+\omega^{2} \epsilon(\boldsymbol{r}) \pm i 0^{+}\right]^{-1}$. Using the SUSY technique [54], $G^{R(A)}$ can be expressed in terms of a path integral over a supervector field. Since a local quantity such as $I\left(x ; x_{0}\right)$ can be expressed in terms of $G^{R(A)}$, upon performing the disorder average, one can express $O\left(x ; x_{0}\right)$ in terms of a functional integral over a supermatrix field $Q(x)[25,26]$. Upon rescaling $x$ by $4 \xi$ where $\xi=\pi \nu D_{0}$ with $\nu$ the density of states per unit length, we find

$$
\begin{aligned}
& O\left(x ; x_{0}\right)=\int D Q(x) M_{O}(Q(x)) \\
& \quad \times \begin{cases}W\left(Q_{L}, Q(x) ; \tilde{L}-x\right) \tilde{W}\left(Q(x), \Lambda ; x, x_{0}\right), & x>x_{0}, \\
\tilde{W}\left(Q_{L}, Q(x) ; \tilde{L}-x, x_{0}-x\right) W(Q(x), \Lambda ; x), & x<x_{0},\end{cases}
\end{aligned}
$$

with $\tilde{L}=L /(4 \xi)$. Different observables $O$ differ only in the functional $M_{O}[Q(x)]$ and in the boundary constraint $Q(L)=$ $Q_{L}[Q(0)$ is a constant matrix $\Lambda]$. Here $W$ is the heat kernel and

$$
\begin{aligned}
\tilde{W}\left(Q, Q^{\prime} ; x_{1}, x_{2}\right) \equiv & \int D Q_{-} D Q_{+} e^{\gamma \operatorname{str}\left(Q_{-} Q_{+}\right)} \\
& \times W\left(Q, Q_{+} ; x_{1}-x_{2}\right) W\left(Q_{-}, Q^{\prime} ; x_{2}\right), \\
& \text { for } x_{1}>x_{2} .
\end{aligned}
$$

Here $-\gamma \operatorname{str}\left(Q_{-} Q_{+}\right)$is the action accounting for wave tunneling through the reflector, with $\gamma$ depending on $\omega, \Gamma$ and the cross sectional area, and str represents the supertrace. Using Eq. (8), we find that both $\partial_{x_{0}} O\left(x ; x_{0}<x\right)$ and $\partial_{x_{0}} O\left(x ; x_{0}>x\right)$ vanish [36]. This justifies Eq. (1).

Applying Eq. (8) to the spatial correlator, $\mathcal{Y}\left(x, x^{\prime}\right) \equiv$ $\left\langle\iint d y d y^{\prime} G^{A}\left(\boldsymbol{r}, \boldsymbol{r}^{\prime}\right) G^{R}\left(\boldsymbol{r}^{\prime}, \boldsymbol{r}\right)\right\rangle$, we find that it is the fundamental solution of the generalized diffusion equation, $-\partial_{x} D\left(x ; x_{0}\right) \partial_{x} \mathcal{Y}=\delta\left(x-x^{\prime}\right)$, from which Eq. (6) follows. We also find $D_{\infty}(\lambda)=1-\lambda+\mathcal{O}\left(\lambda^{3}\right)$ for $\lambda \ll 1$ corresponding to weakly localized waves, with $\lambda$ given by Eq. (7) and $\zeta=\xi /(4 \gamma)$. This perturbative expansion of $D_{\infty}$ is exactly the same for samples without a reflector [26], and the presence of a reflector at $x_{0}$ only enters into $\lambda$. The scaling behavior described by Eq. (2) is expected to hold for all $\lambda$. Thus we can apply the results for localized samples without a reflector [26] to the present case. This gives $D_{\infty} \approx e^{-\lambda}$ for $\lambda \gtrsim 1$ and $D\left(x ; x_{0}\right)=D_{0} e^{-\lambda\left(x ; x_{0}\right)}$ in 1D. Solving Eq. (6) gives Eq. (5).

$$
\begin{aligned}
P\left(\ln I\left(x ; x_{0}\right)\right)= & \iint_{0}^{2 \pi} \frac{d \theta_{l} d \theta_{r}}{(2 \pi)^{2}} \iint_{0}^{2 \pi} \frac{d \mu_{l} d \mu_{r}}{(2 \pi)^{2}} \iint_{0}^{\infty} d \lambda_{l} d \lambda_{r} \\
& \times p_{s_{l}}\left(\lambda_{l}\right) p_{s_{r}}\left(\lambda_{r}\right) \delta\left(\ln I\left(x ; x_{0}\right)-\ln I(x,\{\lambda, \theta, \mu\})\right) .
\end{aligned}
$$

Here $I(x,\{\lambda, \theta, \mu\})$ is the expression for intensity in the polar representation [5]. The parameters $\theta_{l(r)}, \mu_{l(r)}$ are uniformly distributed over $[0,2 \pi]$, and $\lambda_{l(r)}$ is distributed according to $p_{s_{l}\left(s_{r}\right)}\left(\lambda_{l(r)}\right)$ [55,56] with $s_{l}=x / \ell\left[s_{r}=\right.$ $(L-x) / \ell]$. The explicit forms of $I(x,\{\lambda, \theta, \mu\})$ and $p_{s_{l}\left(s_{r}\right)}$ are given in the Supplemental Material [36]. From Eq. (10), one may obtain the statistics of any function of intensity. It gives the values $\langle I(0 ; 0(L))\rangle$ in Eq. (5) and $P(\ln I)$ at $x=L / 2$ plotted in Fig. 1(c) and the analytic expression of Eq. (3).

In general, inhomogeneity arise from multiple segments with different scattering strength or a smoothly varying mean free path, and multiple embedded reflectors. In this case, we map $x$ to $\eta=\eta(x)=\int_{0}^{x}\left[d x^{\prime} / \ell\left(x^{\prime}\right)\right]\left[\eta_{L}=\eta(L)\right]$. A generic average quantity assumes the form $O\left(\eta ;\left\{\eta_{i}\right\}\right)$, where $\left\{\eta_{i}\right\}$ are the coordinates of interfaces or reflectors. Using SUSY, we find

$$
\forall j: \partial_{\eta_{j}} O\left(\eta ;\left\{\eta_{i}\right\}\right)=0, \quad \text { if and only if } \eta_{j} \neq \eta .
$$

Thus $O$ is invariant with regard to arbitrary shuffling of interfaces or reflectors, as long as they do not cross $\eta$. This generalizes Eq. (1). Indeed, Eq. (1) has an equivalent form, namely, $\partial_{x_{0}} O\left(x ; x_{0}\right)=0$ if and only if $x_{0} \neq x$, which is a special case of Eq. (11). 

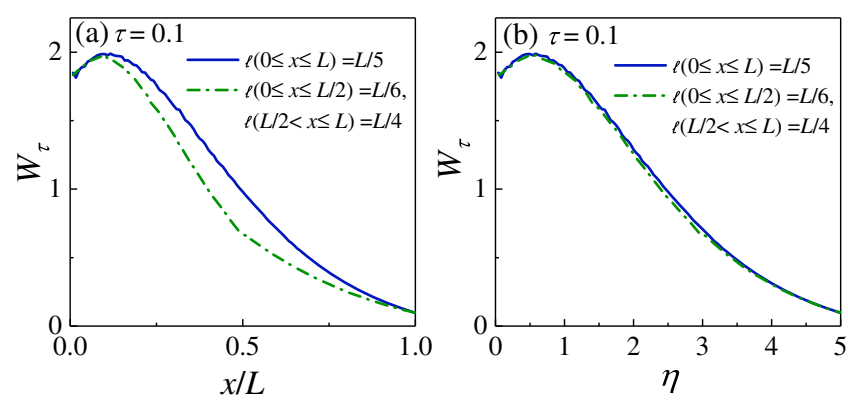

FIG. 4. (a) Simulations of the eigenchannel profile $W_{\tau}$ for two ensembles of 2D diffusive samples. In one ensemble, the sample is composed of two segments of different scattering strength (green dash-dotted curve) while in the other the scattering strength is uniform (blue solid curve). In all samples, $\eta_{L}=5$. (b) Upon mapping $x$ to $\eta(x)$, the profiles of $W_{\tau}$ in the two ensembles collapse to a single curve.

For inhomogeneity arising solely from variations in scattering strength, Eq. (11) implies that the $\eta$ dependence of $O$ is identical to that in a uniformly disordered sample. For $\langle\ln I\rangle$, we find using RMT that $\langle\ln I\rangle=-\eta$. For $\langle I\rangle$, we find using SUSY that $-\partial_{\eta} D(\eta) \partial_{\eta}\langle I\rangle=\delta\left(\eta-\eta^{\prime}\right)$ with $D(\eta)=e^{-\eta\left(\eta_{L}-\eta\right) / \eta_{L}}$, which gives

$\langle I(\eta)\rangle=\langle I(0)\rangle-2[\langle I(0)\rangle-1] \frac{\operatorname{erf}\left(\frac{2 \eta-\eta_{L}}{2 \sqrt{\eta_{L}}}\right)-\operatorname{erf}\left(-\frac{\sqrt{\eta_{L}}}{2}\right)}{\operatorname{erf}\left(\frac{\sqrt{\eta_{L}}}{2}\right)-\operatorname{erf}\left(-\frac{\sqrt{\eta_{L}}}{2}\right)}$.

In Fig. 3, we compare numerical results for intensity statistics in samples with $\eta_{L}=6$ but different variations of $\ell(x)$. The spatial profiles of $\langle\ln I(\eta)\rangle$ and $\langle I(\eta)\rangle$ and the probability distributions of $\ln I$ of any value of $\eta$ are the same in different samples.

The invariance principle for high-dimensional diffusive samples, in which nonuniformity in scattering arises either from an embedded reflector or a spatially varying mean free path, is demonstrated theoretically in the Supplemental Material [36]. In Fig. 4, we compare numerical results for the average longitudinal profile of energy within the sample, denoted $W_{\tau}(x)$, normalized so that $W_{\tau}(x=L)=\tau$ [28,30], in two ensembles consisting of 1000 samples with channel number $N=80$, aspect ratio $2, \eta_{L}=5$, and the scaling conductance $g=N / \eta_{L}=16$. The mean free path is uniform in one ensemble of samples but varies with $x$ in the other. The profiles in two different ensembles overlap upon mapping $x$ to $\eta(x)$.

The invariance principle allows us to shift the focus from the surface to the interior of the sample and from homogeneous to inhomogeneous disorder. We have demonstrated its application to intensity statistics in a general dimension and to controlling the spatial structure of transmission eigenchannels. Our findings open a door to engineering the profiles of wave energy and its flow inside inhomogeneously disordered materials.
This work is supported by the National Science Foundation under Grants No. DMR/-BSF: 1609218 and its successor (A.Z.G.), by National Natural Science Foundation of China Projects No. 11535011, No. 11925507, and No. 11947302 (C. T.), and by MICINN (Spain) under the Project No. PGC2018094684-B-C22 and Subprograma Estatal de Movilidad 2013-2016 under Project No. PRX16/00166 (V. A. G).

*These authors contribute equally to the work. †opar@unizar.es *azriel.genack@qc.cuny.edu

[1] P. Sheng, Introduction to Wave Scattering, Localization, and Mesoscopic Phenomena, 2nd ed. (Springer, Berlin, Germany, 2006).

[2] S. Rotter and S. Gigan, Light fields in complex media: Mesoscopic scattering meets wave control, Rev. Mod. Phys. 89, 015005 (2017).

[3] M. C. W. van Rossum and Th. M. Nieuwenhuizen, Multiple scattering of classical waves: Microscopy, mesoscopy, and diffusion, Rev. Mod. Phys. 71, 313 (1999).

[4] E. Akkermans and G. Montambaux, Mesoscopic Physics of Electrons and Photons (Cambridge University Press, Cambridge, England, 2007).

[5] P. A. Mello and N. Kumar, Quantum Transport in Mesoscopic Systems: Complexity and Statistical Fluctuations (Oxford University Press, Oxford, 2004).

[6] E. Abrahams, P. W. Anderson, D. Licciardello, and T. V. Ramakrishnan, Scaling Theory of Localization: Absence of Quantum Diffusion in Two Dimensions, Phys. Rev. Lett. 42, 673 (1979).

[7] O. N. Dorokhov, Transmission coefficient and the localization length of an electron in $\mathrm{N}$ bound disordered chains, Pis'ma Zh. Eksp. Teor. Fiz. 36, 259 (1982) [JETP Lett. 36, 318 (1982)].

[8] P. A. Mello, P. Pereyra, and N. Kumar, Macroscopic approach to multichannel disordered conductors, Ann. Phys. (N.Y.) 181, 290 (1988).

[9] A. Einstein, Investigations on the Theory of the Brownian Movement (Courier Corporation, Mineola, New York, 1956).

[10] S. Chandrasekhar, Radiative Transfer (Dover, New York, 1960).

[11] P. W. Anderson, Absence of diffusion in certain random lattices, Phys. Rev. 109, 1492 (1958).

[12] M. E. Gertsenshtein and V. B. Vasil'ev, Waveguides with random inhomogeneities and Brownian motion in the Lobachevsky plane, Teor. Veroyatn Primen, 4, 424 (1959) [Theory Probab. Appl. 4, 391 (1959)].

[13] S. L. Jacques, Optical properties of biological tissues: A review, Phys. Med. Biol. 58, R37 (2013).

[14] Deep Imaging in Tissue and Biomedical Materials Using Linear and Nonlinear Optical Methods, edited by L. Shi and R. R. Alfano (Pan Stanford Publishing Pte Ltd, Singapore, 2017).

[15] B. Sklar, Rayleigh fading channels in mobile digital communication systems. I. Characterization, IEEE Commun. Mag. 35, 90 (1997). 
[16] Yu. V. Nazarov, Multiple Scattering of Classical Waves: Microscopy, Mesoscopy, and Diffusion, Phys. Rev. Lett. 73, 134 (1994).

[17] L. Y. Zhao, C. Tian, Y. P. Bliokh, and V. Freilikher, Controlling transmission eigenchannels in random media by edge reflection, Phys. Rev. B 92, 094203 (2015).

[18] A. A. Balandin, Thermal properties of graphene and nanostructured carbon materials, Nat. Mater. 10, 569 (2011).

[19] N. Li, J. Ren, L. Wang, G. Zhang, P. Hänggi, and B. Li, Phononics: Manipulating heat flow with electronic analogs and beyond, Rev. Mod. Phys. 84, 1045 (2012).

[20] S. Lepri, R. Livi, and A. Politi, Thermal conduction in classical low-dimensional lattices, Phys. Rep. 377, 1 (2003).

[21] R. Schittny, A. Niemeyer, F. Mayer, A. Naber, M. Kadic, and M. Wegener, Invisibility cloaking in light-scattering media, Laser Photonics Rev. 10, 382 (2016).

[22] X. Huang, I. H. El-Sayed, W. Qian, and M. A. El-Sayed, Cancer cell imaging and photothermal therapy in the nearinfrared region by using gold nanorods, J. Am. Chem. Soc. 128, 2115 (2006).

[23] P. A. Mello, Z. Shi, and A. Z. Genack, Connection between wave transport through disordered 1D waveguides and energy density inside the sample: A maximum-entropy approach, Physica (Amsterdam) 74E, 603 (2015).

[24] X. Cheng, X. Ma, and M. Yépez, A. Z. Genack, and P. Mello, Single-parameter scaling and maximum entropy inside disordered one-dimensional systems: Theory and experiment, Phys. Rev. B 96, 180203(R) (2017).

[25] C. Tian, Supersymmetric field theory of local light diffusion in semi-infinite media, Phys. Rev. B 77, 064205 (2008).

[26] C. Tian, S. K. Cheung, and Z. Q. Zhang, Local Diffusion Theory for Localized Waves in Open Media, Phys. Rev. Lett. 105, 263905 (2010).

[27] I. M. Vellekoop and A. P. Mosk, Universal Optimal Transmission of Light Through Disordered Materials, Phys. Rev. Lett. 101, 120601 (2008).

[28] M. Kim, Y. Choi, C. Yoon, W. Choi, J. Kim, Q.-H. Park, and W. Choi, Maximal energy transport through disordered media with the implementation of transmission eigenchannels, Nat. Photonics 6, 581 (2012).

[29] A. P. Mosk, A. Lagendijk, G. Lerosey, and M. Fink, Controlling waves in space and time for imaging and focusing in complex media, Nat. Photonics 6, 283 (2012).

[30] M. Davy, Z. Shi, J. Park, C. Tian, and A. Z. Genack, Universal structure of transmission eigenchannels inside opaque media, Nat. Commun. 6, 6893 (2015).

[31] S. M. Popoff, A. Goetschy, S. F. Liew, A. D. Stone, and H. Cao, Coherent Control of Total Transmission of Light Through Disordered Media, Phys. Rev. Lett. 112, 133903 (2014).

[32] A. Lagendijk, R. Vreeker, and P. de Vries, Influence of internal reflection on diffusive transport in strongly scattering media, Phys. Lett. 136A, 81 (1989).

[33] J. X. Zhu, D. J. Pine, and D. A. Weitz, Internal reflection of diffusive light in random media, Phys. Rev. A 44, 3948 (1991).

[34] J. H. Li, A. A. Lisyansky, T. D. Cheung, D. Livdan, and A.Z. Genack, Transmission and Surface Intensity Profiles in Random Media, Europhys. Lett. 22, 675 (1993).
[35] R. H. J. Kop, P. de Vries, R. Sprik, and A. Lagendijk, Fluctuation-Dissipation Theorems and Entropy Production in Relaxational Systems, Phys. Rev. Lett. 79, 4369 (1997).

[36] See the Supplemental Material at http://link.aps.org/ supplemental/10.1103/PhysRevLett.124.057401 for selfcontained discussion of technical aspects of the experiment and of SUSY and RMT, and of the relationship of the theory to measurements, which includes Refs. [37-50].

[37] A. A. Chabanov, M. Stoytchev, and A. Z. Genack, Statistical approach to photon localization, Nature (London) 404, 850 (2000).

[38] B. Rejaei, Equivalence of the transmission-eigenvalue density in supersymmetric and scaling theories of disordered wires without time-reversal symmetry, Phys. Rev. B 53, R13235 (1996).

[39] X. J. Cheng, C. S. Tian, and A. Z. Genack, Transmission eigenvalues in random media with surface reflection, Phys. Rev. B 88, 094202 (2013).

[40] A. D. Mirlin, A. Müller-Groeling, and M. R. Zirnbauer, Conductance fluctuations of disordered wires: Fourier analysis on supersymmetric spaces, Ann. Phys. (N.Y.) 236, 325 (1994).

[41] S. Helgason, Differential Geometry, Lie Groups, and Symmetric Space (Academic Press, New York, 1978).

[42] F. A. Berezin, Introduction to Superanalysis, MPAM Vol. 9 (Springer Netherlands, Dordrecht, 1987).

[43] C. Tian, Hydrodynamic and field-theoretic approaches to light localization in open media, Physica (Amsterdam) 49E, 124 (2013).

[44] C. Tian, A. Kamenev, and A. Larkin, Ehrenfest time in the weak dynamical localization, Phys. Rev. B 72, 045108 (2005).

[45] C. Hainaut, I. Manai, and J.-F. Clément, J.-C. Garreau, P. Szriftgiser, G. Lemarié, N. Cherroret, D. Delande, and R. Chicireanu, Controlling symmetry and localization with an artificial gauge field in a disordered quantum system, Nat. Commun. 9, 1382 (2018).

[46] A. Ossipov and V. E. Kravtsov, T-duality in supersymmetric theory of disordered quantum systems, Phys. Rev. B 73, 033105 (2006).

[47] C. W. J. Beenakker, Random-matrix theory of quantum transport, Rev. Mod. Phys. 69, 731 (1997).

[48] V. I. Mel'nikov, Distribution of resistivity probabilities of a finite disordered system, Pis'ma Zh. Eksp. Teor. Fiz. 32, 244 (1980) [JETP Lett. 32, 225 (1980)].

[49] A. Peña, A. Girschik, F. Libisch, S. Rotter, and A. A. Chabanov, The single-channel regime of transport through random media, Nat. Commun. 5, 3488 (2014).

[50] R. Pierrata, P. Ambichl, S. Gigan, A. Haberb, R. Carminati, and S. Rotter, Invariance property of wave scattering through disordered media, Proc. Natl. Acad. Sci. U.S.A. 111, 17765 (2014).

[51] B. A. van Tiggelen, A. Lagendijk, and D. S. Wiersma, Reflection and Transmission of Waves Near the Localization Threshold, Phys. Rev. Lett. 84, 4333 (2000).

[52] N. Cherroret and S. E. Skipetrov, Microscopic derivation of self-consistent equations of Anderson localization in a disordered medium of finite size, Phys. Rev. E 77, 046608 (2008). 
[53] A. G. Yamilov, R. Sarma, B. Redding, B. Payne, H. Noh, and $\mathrm{H}$. Cao, Position-dependent diffusion of light in disordered waveguides, Phys. Rev. Lett. 112, 023904 (2014).

[54] K. B. Efetov, Supersymmetry in Disorder and Chaos (Cambridge University, Cambridge, England, 1997).
[55] V. A. Gopar and R. A. Molina, Controlling conductance statistics of quantum wires by driving ac fields, Phys. Rev. B 81, 195415 (2010).

[56] I. Kleftogiannis, I. Amanatidis, and V. A. Gopar, Conductance through disordered graphene nanoribbons: Standard and anomalous electron localization, Phys. Rev. B 88, 205414 (2013). 